\title{
Matrix models, topological strings, and supersymmetric gauge theories
}

\section{Citation}

Dijkgraaf, Robbert, and Cumrun Vafa. 2002. "Matrix Models, Topological Strings, and Supersymmetric Gauge Theories." Nuclear Physics B 644 (1-2): 3-20. https://doi.org/10.1016/ s0550-3213(02)00766-6.

\section{Permanent link}

http://nrs.harvard.edu/urn-3:HUL.InstRepos:41385086

\section{Terms of Use}

This article was downloaded from Harvard University's DASH repository, and is made available under the terms and conditions applicable to Other Posted Material, as set forth at http:// nrs.harvard.edu/urn-3:HUL.InstRepos:dash.current.terms-of-use\#LAA

\section{Share Your Story}

The Harvard community has made this article openly available.

Please share how this access benefits you. Submit a story.

Accessibility 
hep-th/0206255

HUTP-02/A028

ITFA-2002-22

\title{
Matrix Models, Topological Strings, and Supersymmetric Gauge Theories
}

\author{
Robbert Dijkgraaf \\ Institute for Theoretical Physics \& \\ Korteweg-de Vries Institute for Mathematics \\ University of Amsterdam \\ 1018 TV Amsterdam, The Netherlands \\ and \\ Cumrun Vafa \\ Jefferson Physical Laboratory \\ Harvard University \\ Cambridge, MA 02138, USA
}

\begin{abstract}
We show that B-model topological strings on local Calabi-Yau threefolds are large $N$ duals of matrix models, which in the planar limit naturally give rise to special geometry. These matrix models directly compute F-terms in an associated $\mathcal{N}=1$ supersymmetric gauge theory, obtained by deforming $\mathcal{N}=2$ theories by a superpotential term that can be directly identified with the potential of the matrix model. Moreover by tuning some of the parameters of the geometry in a double scaling limit we recover $(p, q)$ conformal minimal models coupled to $2 \mathrm{~d}$ gravity, thereby relating non-critical string theories to type II superstrings on Calabi-Yau backgrounds.
\end{abstract}

June, 2002 


\section{Introduction}

Large $N$ limits of $U(N)$ gauge theories have been a source of inspiration in physics, ever since 't Hooft introduced the idea [1]. In particular the large $N$ limit of gauge theories should be equivalent to some kind of closed string theory. The first contact this idea had with string theory was in the context of non-critical bosonic strings described by $c \leq 1$ conformal field theories coupled to two-dimensional gravity. It was found that by taking a "double scaling limit" of $N \times N$ matrix models, where one send $N$ to infinity while at the same time going to some critical point, one ends up with non-critical bosonic strings [2, 23]. This relation between gauge systems and strings was not exactly in the sense that 't Hooft originally suggested for the large $N$ expansion, as it involved a double scaling limit. In particular, before taking this limit the matrix model would not have a string dual, whereas according to 't Hooft's general idea one would have expected it to have.

In one context this was remedied by a different kind of matrix model introduced by Kontsevich [4], where without taking a double scaling limit one finds an equivalence between a matrix model and non-critical string theory. In particular the amplitudes of the topological string observables introduced in [5] are directly computed by these matrix integrals. This duality was in the same spirit as 't Hooft's general idea and can be seen as a low-dimensional example of a holographic correspondence.

More recently large $N$ dualities have come back in various forms. In the context of M-theory a large $N$ matrix formulation was advanced [6]. Since here an unconventional large $N$ limit is involved, this again was not quite in the same spirit as 't Hooft's idea, much as the double scaling limit of matrix models in the context of non-critical strings is not. However, the AdS/CFT correspondence [7] is in the same spirit as 't Hooft's original proposal in that one did not have to take a particular limit to obtain an equivalence. Another example of such a strict large $N$ duality is the relation between Chern-Simons gauge theory and A-model topological strings [8] where one also does not have to take any particular limit for the equivalence to hold.

The main aim of this paper is to develop a mirror version of this last duality [8]. We find matrix models that are dual to B-model topological strings on Calabi-Yau threefolds. This is again in the same spirit as 't Hooft, as one does not take a double scaling limit. We will show in particular that the special geometry of Calabi-Yau threefolds that solves the B-model at tree level emerges naturally from the dynamics of the eigenvalues of the matrix model. 
However, even though it is not required, one can also consider a double scaling limit of this setup and obtain a specific class of Calabi-Yau manifolds that are dual to doublescaled matrix models. In this sense we are enlarging the original equivalence of double scaling limits of matrix models with string theory to an equivalence of all matrix models with some kind of closed string theory, without any need to take a double scaling limit. In particular our result shows that studying strings on non-compact Calabi-Yau spaces provides a unifying approach to all matrix model descriptions.

Furthermore, it turns out that one can embed these large $N$ dualities in the context of type IIB superstrings [9], a relation that was further explored in [10, 11, 12, 13, 14, 15]. In the context of this embedding one obtains a dictionary in which the planar limit of the matrix models is seen to compute superpotentials for certain $\mathcal{N}=1$ supersymmetric gauge theories, where the potential of the matrix model gets mapped to the superpotential for an adjoint scalar of an $\mathcal{N}=1$ theory.

The organization of this paper is as follows: In section 2 we propose the large $N$ conjecture for topological strings with matrix models after reviewing the various geometrical ingredients. In section 3 we pass this conjecture through some highly non-trivial checks, and in particular check it at the planar limit. In section 4 we discuss some generalizations of this conjecture and its connections with non-critical bosonic strings coupled to gravity and the double scaling limit. We also discuss the meaning of the double scaling limit from the viewpoint of type IIB superstrings.

\section{Large $N$ Topological String Conjectures}

In this section we formulate a general class of large $N$ conjectures involving B-model topological strings and certain two-dimensional topological gauge theories. The idea is to consider the mirror of the large $N$ conjecture of [8], which relates large $N$ ChernSimons theory on $S^{3}$ with A-model topological strings on the resolved conifold. This duality involves Calabi-Yau threefold transitions where a 3-cycle with branes wrapped over it shrinks and a 2-cycle grows without any branes wrapped over it. A worldsheet derivation of this duality has been recently presented in [16]. Moreover this has been generalized to a large class of Calabi-Yau threefolds [17] which has been further studied in [18 leading to development of powerful methods to compute all loop A-model topological string amplitudes [19,20]. 
As suggested in [8] the mirror of A-model transitions dualities should also exist, namely we can consider 2-cycles with branes wrapped over them, undergoing transitions where they shrink and are replaced by 3-cycles without any branes. A large class of such Calabi-Yau transitions were considered in [10, 13, [1, 12, 14] in the context of embedding such B-model dualities in type IIB superstrings. However, the duality between gauge theory and the topological B-model itself, which is implicit in these works, has not been studied. The aim of this section is to elaborate on these topological B-model/large $N$ gauge theory dualities.

\subsection{Geometry of the generalized conifold transition}

Instead of being general we consider a special class of such transitions, studied in [10], and discuss its topological lift. The situation considered in [10 involved a string theory realization of $\mathcal{N}=2$ supersymmetric $U(N)$ gauge theory, deformed to $\mathcal{N}=1$ theory by addition of a tree-level superpotential $\operatorname{Tr} W(\Phi)$, which we take to be a general polynomial of degree $n+1$ of the adjoint field $\Phi$. The Calabi-Yau geometry relevant for this was studied in [21] and corresponds to considering the blowup of the local threefold given as a hypersurface

$$
u v+y^{2}+W^{\prime}(x)^{2}=0
$$

The blow up takes place at the critical points of $W$, i.e. at $W^{\prime}(x)=0$. Such transitions lead to a geometry that contains $n$ blown up $\mathbf{P}^{1}$ 's which are all in the same homology class. So in the resolved geometry we can find $n$ isolated rational curves.

More precisely, the resolved singularity can be obtained by starting with the bundle $\mathcal{O}(0) \oplus \mathcal{O}(-2)$ over $\mathbf{P}^{1}$. This is the normal bundle to rational curve in $K 3 \times \mathbf{C}$ and corresponds to $\mathcal{N}=2$ supersymmetry. Let us denote the sections of the normal bundle by $\phi_{0}, \phi_{1}$. These sections are respectively 0 -forms and 1 -forms on the $\mathbf{P}^{1}$. The field $\phi_{0}$ corresponds to the adjoined valued Higgs field $\Phi$ in the $\mathcal{N}=2 \mathrm{SYM}$ theory.

Now the inclusion of the superpotential $W$ should give $n$ isolated $\mathbf{P}^{1}$ 's at the critical values $W^{\prime}\left(\phi_{0}\right)=0$. This is achieved by the following transition function. If $z$ and $z^{\prime}$ are the coordinates on the northern and southern hemispheres of the $\mathbf{P}^{1}$, then the resolution is given by relating the patches $\left(z, \phi_{0}, \phi_{1}\right)$ and $\left(z^{\prime}, \phi_{0}^{\prime}, \phi_{1}^{\prime}\right)$ by

$$
\begin{aligned}
\phi_{0}^{\prime} & =\phi_{0}, \\
z^{\prime} \phi_{1}^{\prime} & =z \phi_{1}+W^{\prime}\left(\phi_{0}\right), \\
z^{\prime} & =1 / z .
\end{aligned}
$$


To relate it to the geometry given by (2.1) we make the identifications

$$
\begin{aligned}
& x=\phi_{0}, \\
& u=2 \phi_{1}^{\prime}, \\
& v=2 \phi_{1}, \\
& \omega=z^{\prime} \phi_{1}^{\prime}, \\
& y=i\left(2 \omega-W^{\prime}\left(\phi_{0}\right)\right),
\end{aligned}
$$

Here we have introduced another variable $\omega$ in terms of which the geometry would have been given by

$$
u v-4 \omega^{2}+4 \omega W^{\prime}(x)=0 .
$$

This makes a natural connection with objects that will be introduced in the next section.

If we now distribute $N$ D5 branes wrapped over these $S^{2}$ 's and filling the spacetime, this corresponds to a choice of the vacuum in the corresponding $\mathcal{N}=1$ supersymmetric gauge theory, where the distribution of the branes among the $n$ critical points corresponds to distributing the $N$ eigenvalues of $\Phi$ among the $n$ classical values. At large $N$ gaugino condensation takes place and this leads to a geometric transition in which the $S^{2}$ 's are all blown down and replaced by "blown up" $S^{3}$ 's. This results in the geometry [10]

$$
u v+y^{2}+W^{\prime 2}(x)+f(x)=0,
$$

where $f(x)$ is a polynomial of degree $n-1$, whose precise coefficients depend on the distribution of the $N$ eigenvalues among the $n$ critical points.

In 150 it has been shown that, if one choses $W(x)$ to be of degree $N+1$ and if all the critical points are equally occupied, the $\mathcal{N}=2$ Seiberg-Witten geometry can be recovered by considering the limit $W \rightarrow \epsilon W$ as $\epsilon \rightarrow 0$.

Note in particular the identification of $x$ with the eigenvalues of $\Phi$. This is rather natural from the equation (2.1). In particular if $W=0$ then we have an $A_{1}$ geometry formed as a hypersurface in $(u, v, y)$ space, where we have wrapped a $D 5$ brane around the blowup sphere. The transverse position of the $D 5$ brane along the $x$ direction would correspond to changing the vev for $\Phi$. Having a non-trivial fibration of the $A_{1}$ geometry over the $x$-plane dictated by (2.1) gives rise to the superpotential $W(\Phi)$, as reviewed above. 
We will be interested in the B-model topological string on the deformed geometry given by equation (2.5). The genus zero prepotential is determined by period integrals of the holomorphic $(3,0)$ form

$$
\Omega=\frac{d x \wedge d u \wedge d v}{\sqrt{u v+W^{\prime}(x)^{2}+f(x)}} .
$$

This non-compact Calabi Yau has $n$ compact three-cycles $A_{i}$ that correspond to the $n$ "blown-up" $S^{3}$ and there are $n$ canonically conjugated cycles $B_{i}$ that are non-compact and have the topology of a three-ball. The usual special geometry relations determine the tree-level prepotential $\mathcal{F}_{0}\left(S_{i}\right)$ by the periods

$$
\begin{aligned}
S_{i} & =\int_{A_{i}} \Omega, \\
\frac{\partial \mathcal{F}_{0}}{\partial S_{i}} & =\int_{B_{i}} \Omega .
\end{aligned}
$$

Here one should be careful in regulating the periods over the non-compact $B$ cycles [10]. The degree of the deformation $f(x)$, which corresponds to normalizable deformations, is such that these periods can be sensibly defined. In particular their variations with respect to the variation of the coefficients of $f(x)$ are cutoff independent.

As explained in [10] the $A$ and $B$ cycles can be represented as $S^{2}$ fibrations over paths in the complex $x$-plane. After integrating the 3 -form over these $S^{2}$ fibers we are left with the integrals over these curves of the 1 -form

$$
\eta=y d x
$$

Here $y$ is determined by the hyperelliptic curve

$$
y^{2}+W^{\prime}(x)^{2}+f(x)=0 .
$$

We will refer to this curve as the spectral curve. It has $n$ branch cuts that are the projections of the cycles $A_{i}$ onto the $x$-plane. The cycles $B_{i}$ are represented by half-lines that start at the cuts and run to some cut-off point $x=\Lambda$ far away from the branch cuts. 


\subsection{Lifting to topological string dualities}

We now consider lifting these dualities to topological strings. As discussed in [9] the key point is the observation in [22] that topological strings computes superpotential terms of the corresponding gauge theory arising in type II superstrings. In particular the leading planar diagram computes superpotential terms involving the gaugino bilinear field on the gauge theory side, and the $\mathcal{N}=2$ prepotential on the dual gravity side (with some vev's for auxiliary fields, corresponding to turning on fluxes). More generally the topological gravity in the presence of Calabi-Yau 3-fold is the B-model theory studied in [22] and called "Kodaira-Spencer theory of gravity". Thus all we need to do is to specify the gauge theory dual, which should be the gauge theory on the topological branes wrapping the $\mathbf{P}^{1}$ 's. This theory is the reduction of the holomorphic Chern-Simons theory studied in 23 from complex dimension three to complex dimension one, which we now turn to.

First suppose that we have no superpotential, i.e. $W(x)=0$. In this case we simply get the $A_{1}$ geometry times the $x$-plane. We wrap $N$ branes around the $\mathbf{P}^{1}$. In this case the normal directions to the $P^{1}$ correspond to the cotangent bundle and the trivial bundle C associated to $x$. Let us call the Higgs fields in these two direction respectively $\Phi_{1}(z)$ for the cotangent direction and $\Phi_{0}(z)$ for the $x$-direction. The topological theory on the B-brane we obtain in this case is given by the action

$$
S=\frac{1}{g_{s}} \int_{\mathbf{P}^{1}} \operatorname{Tr}\left(\Phi_{1} \bar{D}_{A} \Phi_{0}\right)
$$

where $\Phi_{1}(z)$ is a $U(N)$ adjoint valued $(1,0)$ form on $\mathbf{P}^{\mathbf{1}}, \Phi_{0}(z)$ is an adjoint valued scalar, $A(z)$ is a $U(N)$ holomorphic $(0,1)$ form connection on $\mathbf{P}^{1}$, and $\bar{D}_{A}=\bar{\partial}+[A,-]$. Here $g_{s}$ denotes the string coupling constant. If we turn on the Higgs fields thereby deforming the $\mathbf{P}^{1}$ to a non-holomorphic curve $\mathcal{C}$, this action computes the integral

$$
S=\frac{1}{g_{s}} \int_{Y} \Omega
$$

with $Y$ a 3 -chain connecting $\mathbf{P}^{1}$ and $\mathcal{C}$.

If we turn on the superpotential $W$ by deforming the geometry, the above action changes. This has been studied in [21] by studying the Beltrami differential that deforms the complex structure in the appropriate way, leading (including a minor generalization) to

$$
S_{W}=\frac{1}{g_{s}} \int_{\mathbf{P}^{1}} \operatorname{Tr}\left(\Phi_{1} \bar{D}_{A} \Phi_{0}+W\left(\Phi_{0}\right) \omega\right)
$$


where $W\left(\Phi_{0}\right)$ corresponds to the superpotential and $\omega$ is a $(1,1)$ form which can be taken to correspond to have unit volume on $\mathbf{P}^{1}$.

A consistency check for this action is to note that the equations of motion following from the above action agree with the fact that the classical solutions correspond to holomorphic curves. In particular integrating out $A$ gives

$$
\left[\Phi_{0}, \Phi_{1}\right]=0
$$

so that $\Phi_{0}$ and $\Phi_{1}$ commute, i.e. we can assume they are simultaneously diagonal. Variation with respect to the eigenvalues of $\Phi_{1}$ leads to

$$
\bar{\partial} \Phi_{0}=0
$$

which together with compactness of $\mathbf{P}^{1}$ implies that $\Phi_{0}$ is a constant. Variation with respect to $\Phi_{0}$ gives

$$
\bar{\partial} \Phi_{1}=W^{\prime}\left(\Phi_{0}\right) \omega
$$

which, together with the fact that the integral of $\bar{\partial} \Phi_{1}$ over $\mathbf{P}^{1}$ has to be zero for nonsingular $\Phi_{1}$, leads to

$$
W^{\prime}\left(\Phi_{0}\right)=0=\Phi_{1}
$$

Thus the classical vacua indeed are localized at points $W^{\prime}\left(\Phi_{0}\right)=0$, which describe the positions of the $n \mathbf{P}^{1}$ 's..

In fact the action (2.8) and the resulting quantum theory is rather trivial. In particular $\Phi_{1}$ also appears linearly and can be integrated out exactly, leading to the constraint $\bar{\partial} \Phi_{0}=$ 0 , which leads to the statement that $\Phi_{0}$ is a constant $N \times N$ matrix

$$
\Phi_{0}(z)=\Phi=\text { cnst }
$$

Thus the full action just reduces to its last potential term, which after integration of $\omega$ over $\mathbf{P}^{1}$ leads to the matrix action

$$
S_{W}(\Phi)=\frac{1}{g_{s}} \operatorname{Tr} W(\Phi)
$$

Thus we see that the partition function of the gauge system is equivalent to a simple matrix model! 


\subsection{Operator formalism}

We can give another derivation for the action (2.8) starting directly from the patching functions (2.2) that determine the blown-up geometry. We split the $\mathbf{P}^{1}$ in two hemispheres connected by a long cylinder. We denote the fields on these two patches as $\Phi_{0}, \Phi_{1}$ respectively $\Phi_{0}^{\prime}, \Phi_{1}^{\prime}$. In the case $W=0$ we are simply dealing with a gauged chiral CFT given by an adjoined valued $\beta-\gamma$ system of spin $(1,0)$. The partition function computes the number of holomorphic blocks and this is one on the two-sphere. (Here we are ignoring for a moment the factor coming from the volume of $U(N)$.) In an operator formalism this partition is simply given by pairing the left and right vacuum

$$
Z=\langle 0 \mid 0\rangle=1 \text {. }
$$

Now we want to implement the deformation induced by $W$. From the transition function (2.2) we see that the fields are related in the following way (here we write the fields in coordinates on the cylinder so that factors of $z$ and $z^{\prime}$ are absorbed)

$$
\Phi_{1}^{\prime}=\Phi_{1}+W^{\prime}\left(\Phi_{0}\right) \text {. }
$$

Now there is an obvious operator $U$ that implements this transformation on the Hilbert space. If we define

$$
U=\exp \frac{1}{g_{s}} \oint \operatorname{Tr} W\left(\Phi_{0}(z)\right) d z
$$

(recall that the operator $\Phi_{0}(z)$ is an holomorphic field, so the contour does not matter as long as it encircles the poles) then one easily verifies that

$$
\Phi_{1}^{\prime}=U \Phi_{1} U^{-1} .
$$

Here one uses the fact that the fields $\Phi_{0}$ and $\Phi_{1}$ are canonically conjugated

$$
\Phi_{0}(z) \Phi_{1}(w) \sim \frac{g_{s}}{z-w} .
$$

Therefore in an Hamiltonian formalism the partition function should be given by inserting the transformation $U$ between the left and right vacua

$$
Z=\langle 0|U| 0\rangle \text {. }
$$

This is the familiar way to implement changes in complex structure in the operator formalism of conformal field theory.

In equation (2.10) we have written the deformation of the action in terms of the contour integration or Wilson line $\oint \operatorname{Tr} W\left(\Phi_{0}\right)$. Alternatively, this can be written as a surface integral

$$
U=\exp \frac{1}{g_{s}} \int_{\mathbf{P}^{1}} \operatorname{Tr} W\left(\Phi_{0}\right) \omega,
$$

where the volume form $\omega$ has been localized to a band along the equator of the $\mathbf{P}^{1}$. But, as noted above, one can take any 2-form, as long as it integrates to 1 over the sphere. 


\subsection{Precise Conjecture}

We are now ready to state our conjecture in precise terms. Let $\mathcal{F}_{W, f}\left(g_{s}\right)$ denote the partition function of topological B-model for the Calabi-Yau manifold given by

$$
u v+y^{2}+W^{\prime}(x)^{2}+f(x)=0
$$

where $W$ is a fixed polynomial of degree $n+1$ in $x$ and $f$ is a polynomial of degree $n-1$ in $x$. Let $S_{i}$ denote the integral of the holomorphic 3 -form over the $i$-th $S^{3}$ coming from the $i$-th critical point of $W$. The periods $S_{i}$ will vary as we vary the $n$ coefficients of $f$. Inverting this map, given the variables $S_{i}$ we can find the coefficients of the polynomial $f(x)$ compatible with these periods.

On the gauge theory side we now consider the matrix model given by the action

$$
S_{W}(\Phi)=\frac{1}{g_{s}} \operatorname{Tr} W(\Phi)
$$

We expand this matrix model near the classical vacuum given by partitioning

$$
N=N_{1}+\ldots+N_{n}
$$

and by putting $N_{i}$ eigenvalues of $\Phi$ in the $i$-th critical point of $W$. (Here we use both stable and unstable critical points. In fact, since we work in the holomorphic context, this difference does not really make sense.) Let $\mathcal{F}_{W, N_{i}}\left(g_{s}\right)$ denote the free energy of this matrix model expanded near this classical vacuum. Then the claim is

$$
\mathcal{F}_{W, f}\left(g_{s}\right)=\mathcal{F}_{W, N_{i}}\left(g_{s}\right)
$$

with the understanding that $N_{i} g_{s}=S_{i}$ and, as discussed above, the periods $S_{i}$ fix the coefficients in the polynomial $f(x)$.

A few comments are in order: The matrix model integral is over a holomorphic matrix $\Phi$, i.e. we have integrals of the form $\int d \Phi$ and not like $\int d \Phi d \bar{\Phi}$. This is a general issue in topological B-branest. The world-volume actions, such as the holomorphic ChernSimons action in six dimensions, is a holomorphic function of the field variables. The non-perturbative path-integral should therefore by defined by picking some appropriate contour in the complex field configuration space. Indeed in our two-dimensional example

1 We thank E. Witten for a useful discussion on this point. 
we ended up with a chiral CFT and the path-integral definition of such a theory is rather subtle. One often ends up defining it as a holomorphic square root of a non-chiral theory. For a perturbative computation the situation is much easier. One simply performs the Feynman diagrams as if one was dealing with a real field. Similarly in this case we can effectively treat the matrix $\Phi$ as if it is a real, that is Hermitean, matrix.

Secondly we should note that the matrix integral, in the particular vacuum we end up, has a prefactor of

$$
1 / \operatorname{Vol}\left(U\left(N_{1}\right) \times \cdots \times U\left(N_{n}\right)\right) .
$$

This comes from the fact that for this vacuum $U\left(N_{1}\right) \times \cdots \times U\left(N_{n}\right)$ denotes the unbroken gauge group and we have to mod out by the corresponding volume of the constant gauge transformations. This piece gives, as discussed in [16] the partition function of $c=1$ at self-dual radius. In particular the genus 0 answer will involve

$$
\mathcal{F}_{0}=\sum_{i} \frac{1}{2} S_{i}^{2} \log S_{i}
$$

In embedding in type IIB superstring this leads to the gaugino superpotential $W_{\text {eff }}=$ $\sum_{i} N_{i} \partial \mathcal{F}_{0} / \partial S_{i}+\alpha S_{i}$. (Note that within the type IIB context the parameters $S_{i}$ and $N_{i}$ are independent.) This is a first check on our conjecture. We are now ready to test the above large $N$ conjecture in more detail.

\section{Matrix models and special geometry}

We will first show how our conjecture can be proven in the planar limit using standard manipulations in matrix model technology. A useful reference is for example [3]. We will see how the special geometry of Calabi-Yau's emerges naturally.

\subsection{Matrix integrals in the planar limit}

We consider the one-matrix integral over $N \times N$ Hermitean matrices $\Phi$

$$
Z=\frac{1}{\operatorname{Vol}(U(N))} \int d \Phi \cdot \exp \left(-\frac{1}{g_{s}} \operatorname{Tr} W(\Phi)\right)
$$

with $W(\Phi)$ a polynomial of degree $n+1$. By diagonalizing the matrix $\Phi$ such integrals can be reduced to an integral over the eigenvalues $\lambda_{1}, \ldots, \lambda_{N}$. In this way we obtain

$$
Z=\int \prod_{i} d \lambda_{i} \cdot \Delta(\lambda)^{2} \cdot \exp \left(-\frac{1}{g_{s}} \sum_{i} W\left(\lambda_{i}\right)\right),
$$


where the Vandermonde determinants

$$
\Delta(\lambda)=\prod_{i<j}\left(\lambda_{i}-\lambda_{j}\right)=\operatorname{det}\left(\lambda_{i}^{j-1}\right)
$$

appear from the Jacobian picked up by the diagonalization process. After exponentiating this contribution the effective action for the eigenvalues is given by

$$
S(\lambda)=\frac{1}{g_{s}} \sum_{i} W\left(\lambda_{j}\right)-2 \sum_{i<j} \log \left(\lambda_{i}-\lambda_{j}\right) .
$$

In this way we end up with a collection of $N$ variables $\lambda_{1}, \ldots, \lambda_{N}$ in a potential $W(\lambda)$. These eigenvalues interact through the second term that is a consequence of integrating out the off-diagonal components. This term gives the famous Coulomb repulsion between the eigenvalues. Because of the effective Pauli exclusion principle induced by the Vandermondes it makes the eigenvalues behave as fermions. From this action we see that the equation of motion satisfied by the eigenvalues is

$$
\frac{1}{g_{s}} W^{\prime}\left(\lambda_{i}\right)-2 \sum_{j \neq i} \frac{1}{\lambda_{i}-\lambda_{j}}=0
$$

We will now take the limit $N \rightarrow \infty$ of this system while keeping fixed the 't Hooft coupling

$$
\mu=g_{s} N
$$

In this standard large $N$ limit we will have a continuum of eigenvalues and their density

$$
\rho(\lambda)=\frac{1}{N} \sum_{i} \delta\left(\lambda-\lambda_{i}\right)
$$

becomes a continuous function on the real axis normalized to $\int \rho(\lambda) d \lambda=1$. (In the following $\lambda$ will always denote a real variable, in contrast with the variable $x$ that can be complex). The eigenvalues will fill a domain on the real axis. This domain might consist of several disconnected components known as cuts. In the case of more than one components one speaks of a multi-cut solution. In the present case they are at most $n$ of these cuts. We will denote the corresponding intervals in the complex $x$ plane as $A_{i}, i=1, \ldots, n$. 


\subsection{The spectral curve}

To further analyze the model it will be convenient (and standard practice) to introduce the trace of the resolvent of the matrix $\Phi$

$$
\omega(x)=\frac{1}{N} \operatorname{Tr}\left(\frac{1}{\Phi-x}\right)=\frac{1}{N} \sum_{i} \frac{1}{\lambda_{i}-x}, \quad x \in \mathbf{C}, x \neq \lambda_{i}
$$

This resolvent plays an crucial role in matrix model technology. It also has an interesting physical interpretation. For example, it can be thought of as a loop operator.

By multiplying the equation of motion (3.2) by the factor $1 /\left(\lambda_{i}-x\right)$ and summing over $i$ one obtains the important relation (loop equation) [3]

$$
\omega^{2}(x)-\frac{1}{N} \omega^{\prime}(x)+\frac{1}{\mu} \omega(x) W^{\prime}(x)+\frac{1}{4 \mu^{2}} f(x)=0
$$

where the polynomial $f(x)$ is of degree $n-1$ and is given by

$$
f(x)=\frac{4 \mu}{N} \sum_{i} \frac{W^{\prime}(x)-W^{\prime}\left(\lambda_{i}\right)}{x-\lambda_{i}}
$$

In some sense the function $f(x)$ determines through (3.3) the whole solution of the matrix integral. Since it is polynomial of degree $n-1$ we only have to determine the $n$ unknown coefficients.

In the large $N$ limit the second term in (3.3) can be ignored and the differential equation for $\omega(x)$ becomes an algebraic equation

$$
\omega^{2}(x)+\frac{1}{\mu} \omega(x) W^{\prime}(x)+\frac{1}{4 \mu^{2}} f(x)=0
$$

From this we see that the resolvent $\omega(x)$ in general has a piece that can have branch cuts. This singular part is captured by the function $y(x)$ that we define here as

$$
\begin{aligned}
y(x) & =2 \mu \omega(x)+W^{\prime}(x) \\
& =2 g_{s} \sum_{i} \frac{1}{\lambda_{i}-x}+W^{\prime}(x) .
\end{aligned}
$$

In terms of the variables $(x, y)$ the relation (3.5) associated to the matrix model now takes the form

$$
y^{2}-W^{\prime}(x)^{2}+f(x)=0 .
$$


This has an interpretation as a hyperelliptic curve in the $(x, y)$-plane. We immediately recognize this curve as the curve (2.7) that determined the periods and thereby the treelevel topological string on the deformed Calabi-Yau geometry. In fact, it is even more natural to identify directly the parametrization of the curve in terms of $(x, \omega)$ in (3.5) with equation (2.4)of section 2. From this we see that we should identify $\omega$ with the field $z \Phi_{1}$.

Note that the function $y(x)$ naturally appears from the dynamics of the eigenvalues. It is given by the variation of the action (3.1) with respect to a particular eigenvalue $\lambda$

$$
y(\lambda)=g_{s} \frac{\partial S}{\partial \lambda} .
$$

In fact, from this we see that the quantity $y(x)$ has an elegant physical interpretation. Being the derivative of the potential energy, it equals the force on an eigenvalue if it tries to go away from its stationary position and moves into the complex $x$-plane.

\subsection{Filling fractions and periods}

We now want to evaluate the matrix integral around a particular stationary point where particular fractions of the eigenvalues cluster around the different critical points. Around such a multi-cut configuration it makes sense to make a perturbative expansion of the matrix integral using large $N$ techniques. It is the contribution from one of these saddle points that we are after.

Consider such a multi-cut solution. The filling fractions $N_{i} / N$, i.e. the relative number of eigenvalues around each critical point, are given by the integrals

$$
N_{i} / N=\int_{A_{i}} \rho(\lambda) d \lambda
$$

Here the $A_{i}$ are the cuts in the complex $x$-plane.

Now an important standard result is that the density of eigenvalues is given by the jump of the resolvent $\omega(x)$ across the cut

$$
\rho(\lambda)=\frac{1}{2 \pi i}(\omega(\lambda+i 0)-\omega(\lambda-i 0)) .
$$

Since only the singular piece contributes, we can also write this relation as

$$
\rho(\lambda)=\frac{\mu}{\pi i}(y(\lambda+i 0)-y(\lambda-i 0)) .
$$


Therefore we can compute the fraction of eigenvalues in a specific cut by doing a contour integral around the cut. In this way we find that the quantities $S_{i}=g_{s} N_{i}$ are given by the period integrals around the cut, that is the periods on the Riemann surface

$$
S_{i}=\frac{1}{2 \pi i} \oint_{A_{i}} y(x) d x
$$

Here we make contact with the period integrals (2.6) as obtained in the topological Bmodel computation. This is the first half of the derivation of the genus zero part of our conjecture.

In order to complete the derivation, we now need to compute the change in the free energy $\mathcal{F}_{0}\left(S_{i}\right)$ if we vary the filling fractions $S_{i}$ by adding an eigenvalue to the cuts

$$
\Delta S_{i}=\frac{1}{g_{s}} \Delta N_{i}
$$

This change in the action is given by the work done by the force $F(x)$ acting on an eigenvalue if we move this eigenvalue from one branch to infinity. We have seen in (3.8) that this force is given by

$$
F(x)=\frac{1}{g_{s}} y(x)
$$

So the variation of the free energy is computed in terms of the action $\int F(x) d x$, that is by integrating the one-form $y(x) d x$ along one of the $B$-cycles to the cut-off point $x=\Lambda$. We therefore immediately find the special geometry relation

$$
\frac{\partial \mathcal{F}_{0}}{\partial S_{i}}=\int_{B_{i}} y(x) d x
$$

expressing the $B$-periods in terms of the $A$-periods through the free energy $\mathcal{F}_{0}(S)$. Together (3.10) and (3.11) give the precise match with the Calabi-Yau geometry. This concludes the derivation of the planar version of our conjecture relating the matrix model to the topological B-model on the deformed Calabi-Yau.

As we have mentioned, for a given potential $W(x)$ the function $f(x)$ that deforms the singular Calabi-Yau and determines the solution of the matrix model can be expressed in terms of the periods $S_{i}$ and vice versa. In fact, using definition (3.4) we can give a useful relation valid in terms of the prepotential $\mathcal{F}_{0}$. If one parametrizes the potential as

$$
W(x)=\sum_{k=0}^{n+1} u_{k} x^{k}
$$


and the deformation as

$$
f(x)=\sum_{k=0}^{n-1} 4 \mu b_{k} x^{k},
$$

then by plugging definition (3.4) in the matrix integral we obtain

$$
b_{k}=(k+2) u_{k+2}+\sum_{j=k+2}^{n+1} j u_{j} \frac{\partial \mathcal{F}_{0}\left(u_{i}, S_{i}\right)}{\partial u_{j-k-2}} .
$$

Note that if we introduce Virasoro operators $L_{k}=\sum_{j} j u_{j} \partial / \partial u_{j+k}$ this equation can be written as 24]

$$
b_{k}=(k+2) u_{k+2}+L_{-k-2} \mathcal{F}_{0}, \quad k=0, \cdots, n-1 .
$$

\subsection{Higher genus}

Can one extend the derivation of our conjecture to higher genera? One possibility might be to use the loop equations that are derived by taking the expectation value of expression (3.3) for the resolvent $\omega(x)$

$$
\left\langle\omega^{2}(x)\right\rangle+\frac{1}{\mu}\langle\omega(x)\rangle W^{\prime}(x)+\frac{1}{4 \mu^{2}} f(x)=0,
$$

where $f(x)$ is now given by the expectation value of expression (3.4). These loop equations have been studied for multi-cut solutions, for example in [25], and one can try to solve them order by order in the string coupling constant $g_{s}$. In principle these equations give recursion relations that relate the higher genus amplitudes in terms of the tree-level free energy. It would be very interesting to see if these equations are directly related to the equations of motion for Kodaira-Spencer string field theory [22]. This is not completely unlikely since collective field theories for the eigenvalue densities are known to have a similar form [26], and morally the connection between topological strings and matrix models should go along these lines.

It might be interesting to also briefly discuss the case of the pure conifold, here given by the quadratic superpotential $W(x)=x^{2}$. The large $N$ dual is from our point of view just the Gaussian matrix model

$$
Z=\frac{1}{\operatorname{Vol}(U(N))} \int d \Phi \cdot e^{-\frac{1}{g_{s}} \operatorname{Tr} \Phi^{2}} .
$$

Since the integral is trivial, the only contribution comes from the normalization factor

$$
\frac{1}{\operatorname{Vol}(U(N))} \sim \prod_{k=1}^{N-1} k !
$$


which has been shown to reproduce the all genus answer for the B-model on the conifold in the $1 / N$ expansion [16]. In this case the spectral curve is given by

$$
y^{2}-x^{2}+\mu=0
$$

and the eigenvalue density

$$
\rho(\lambda)=\sqrt{\lambda^{2}-\mu}
$$

is Wigner's famous semi-circle distribution. In the eigenvalue basis the all genus answer is alternatively obtained by the method of orthogonal polynomials which indeed gives [27]

$$
\int \prod_{i} d \lambda_{i} \cdot \Delta(\lambda)^{2} \cdot e^{-\frac{1}{g_{s}} \sum_{i} \lambda_{i}^{2}} \sim \prod_{k=1}^{N-1} k !
$$

\subsection{Domain walls and eigenvalue tunneling}

There is an interesting relation that directly connects the D-branes in the type II string theory and the behaviour of the eigenvalues in the matrix models. In the "old days" it was pointed out by Shenker [28] that the characteristic non-perturbative effect observed in matrix models was the tunneling of eigenvalues and this was an effect of strength $1 / g_{s}$. This remark in some sense anticipated the importance of D-branes. Here we can connect the two effects.

In the type IIB theory on the resolved geometry we can consider D5-branes wrapped around an $S^{3}$ that interpolates between two $S^{2}$. Such an object manifest itself as a domain wall in the four uncompactified spacetime dimensions. After the geometric transition such a D5-brane will connect two three-cycles. It will describe a process where one unit of RR flux is transported. That is, the RR flux in one $S^{3}$ is decreased by one unit and the flux in another $S^{3}$ is increased by one. Since the space-time superpotential is given by

$$
W_{e f f}=\sum_{i}\left(N_{i} \frac{\partial \mathcal{F}_{0}}{\partial S_{i}}+\alpha S_{i}\right)
$$

the tension of a domain wall transferring flux from the $i$-th to the $j$-th cycle is given by

$$
T=\frac{\partial \mathcal{F}_{0}}{\partial S_{i}}-\frac{\partial \mathcal{F}_{0}}{\partial S_{j}}=\int_{B_{i j}} y(x) d x
$$

We now recognize this as the instanton action in the matrix model of an eigenvalue tunneling from the cut $A_{i}$ to the cut $A_{j}$ along the path $B_{i j}$. 


\section{Double scaling limits and other further generalizations}

Given that we have found a natural stringy interpretation of ordinary matrix model, one could ask what is the meaning of the double scaling limit in the context of the old matrix model [3]. Following that limit on the gravity side for the single matrix model leads to the Calabi-Yau geometry

$$
H=u v+y^{2}+x^{2 m+1}+\text { deformations }=0
$$

as corresponding to the $(2,2 m+1)$ bosonic minimal model coupled to two-dimensional gravity. The deformations correspond to the $m$ observables of the $(2,2 m+1)$ model. In fact for generic deformations of this geometry there are $m$ A-cycles and $m$ B-cycles and we thus can choose $m$ independent parameters to parametrize these deformations. The infinitesimal deformations which map this geometry to the deformations of the $(2,2 m+1)$ models are of the form

$$
u v+y^{2}+x \prod_{i=1}^{m}\left(x-\epsilon_{i}\right)^{2}=0
$$

where $\epsilon_{i}$ are related to the deformation of the $(2,2 m+1)$ model with primary fields. For example, the case of pure $2 \mathrm{~d}$ gravity, i.e. the $(2,3)$ model has only one observable and in that case we have $\epsilon=\mu^{1 / 2}$. In the usual matrix model this is obtained by considering a quartic superpotential $W(x)$. Let us take that to be an even function of $x$. Then there are three critical points, including one at $x=0$. Upon deformation by $f(x)$ the critical points can split. If we put all the $N$ eigenvalues at the well corresponding to $x=0$ then only the $x=0$ double point splits and the other two do not split. The double scaling limit corresponds to taking the limit where one of the double points reaches one pair of doubles and the other reaches the other pair. Taking the limit where the geometry is localized near one set of triple zeros we obtain the $(2,3)$ local geometry given in (4.1). Embedding this kind of theory in type IIB strings gives exactly the kind of $N \rightarrow \infty$ dualities proposed recently in [19] which relate certain limits of gauge systems with type IIB strings on Calabi-Yau geometries without fluxes.

Note that this map to topological B-model is in perfect accord with the fact that the bosonic strings have a hidden $\mathcal{N}=2$ superconformal symmetry [29], which in this case we can identify with the superconformal theory on the above Calabi-Yau threefold. As a check note that, if we only turn on the cosmological constant, then the genus $g$ answer for 
topological B-model scales as the holomorphic threeform $\Omega$ to the power of $2-2 g$ [22]. For the $(2,3)$ model since we have

$$
\Omega=\frac{d u d v d y d x}{d H} \sim \mu^{5 / 4}
$$

So we learn that the free energy of the B-model is given by an expansion of the form

$$
\mathcal{F}=\sum_{g \geq 0} c_{g} g_{s}^{2 g-2} \mu^{\frac{5}{4}(2-2 g)}
$$

for some coefficients $c_{g}$, in perfect accord with the expected answer.

As a further generalization one can consider the topological lift of the models considered in [14]. These involve many $U(N)$ gauge fields with bifundamental matter. In particular for the case of a linear chain of $p-1$ gauge fields one obtains the dual geometry (before blow up)

$$
u v+\prod_{i=1}^{p}\left(y-W_{i}^{\prime}(x)\right)=0 .
$$

where the $W_{i}(x)$ are related to the superpotentials of the corresponding gauge fields. Again a double scaling limit exists which lead to geometries of the form

$$
u v+y^{p}+x^{q}+\text { deformations }=0
$$

This would correspond to the $(p, q)$ bosonic minimal model coupled to gravity. There are $\frac{1}{2}(p-1)(q-1)$ A-cycles for deformations in the above equation, which correspond to the observables of the $(p, q)$ minimal model. As discussed in 13 in this case the reduction of holomorphic 3 -form leads to $p-1$-forms $\eta_{i}$ which naturally get identified with the $p-1$ eigenvalue densities in a $(p-1)$ matrix model. It would be interesting to study these model in more detail and in particular the corresponding multi-matrix model duals.

\subsection{Final remarks}

There are many indications that topological strings on a general target space might be described by some kind of integrable systems. This was originally shown for the $c \leq 1$ topological string theories [30]. More recently in the context of A-model topological strings on non-compact Calabi-Yau this integrability was shown by dualizing to certain observables of Chern-Simons gauge theory on three-manifolds [19,20]. For A-models on compact target spaces evidence has been accumulating in the mathematical literature on Gromov-Witten 
invariants (see for example [31,32]). If we manage to formulate a matrix model dual of the topological string this integrability is in some sense manifest. Both the finite $N$ and the large $N$ matrix models are well-known to give tau-functions of the KP and Toda hierarchies [3,27]. This integrability of matrix models was the underlying reason that noncritical strings with $c \leq 1$ were exactly solvable. Our results indicate that for a large class of non-compact Calabi-Yau manifolds this integrability is present.

Furthermore one should also like to be able to include gravitational descendents within the topological string model. In terms of the B-model we expect that these are given by non-normalizable deformations of the complex structure.

A related issue is the description of the $c=1$ string, which is equivalent to topological strings on the conifold geometry [33]. In a forthcoming paper [34] we will describe how tachyon scattering processes are indeed reproduced in the conifold string theory and can be described by a large $N$ dual gauge system, making contact with [35] and the recent work [36].

\section{Acknowledgements}

We would like to thank F. Cachazo, V. Kazakov, G. Moore and H. Ooguri for valuable discussions. R.D. would like to thank the Harvard Physics Department and the Institute for Advanced Study, Princeton for kind hospitality during part of this work. The research of R.D. is partly supported by FOM and the CMPA grant of the University of Amsterdam, C.V. is partly supported by NSF grants PHY-9802709 and DMS-0074329. 


\section{References}

[1] G. 't Hooft, "A Planar Diagram Theory For Strong Interactions," Nucl. Phys. B 72, 461 (1974).

[2] P. Ginsparg and G. W. Moore, "Lectures On 2-D Gravity And 2-D String Theory," arXiv:hep-th/9304011.

[3] P. Di Francesco, P. Ginsparg and J. Zinn-Justin, "2-D Gravity and random matrices," Phys. Rept. 254, 1 (1995) arXiv:hep-th/9306153

[4] M. Kontsevich, "Intersection Theory On The Moduli Space Of Curves And The Matrix Airy Function," Commun. Math. Phys. 147, 1 (1992).

[5] E. Witten, "On The Structure Of The Topological Phase Of Two-Dimensional Gravity," Nucl. Phys. B 340, 281 (1990)

[6] T. Banks, W. Fischler, S. H. Shenker and L. Susskind, "M theory as a matrix model: A conjecture," Phys. Rev. D 55, 5112 (1997) arXiv:hep-th/9610043.

[7] O. Aharony, S. S. Gubser, J. M. Maldacena, H. Ooguri and Y. Oz, "Large N field theories, string theory and gravity," Phys. Rept. 323, 183 (2000) arXiv:hep-th/9905111.

[8] R. Gopakumar and C. Vafa, "On the gauge theory/geometry correspondence," Adv. Theor. Math. Phys. 3, 1415 (1999) arXiv:hep-th/9811131.

[9] C. Vafa, "Superstrings and topological strings at large N," J. Math. Phys. 42, 2798 (2001) arXiv:hep-th/0008142.

[10] F. Cachazo, K. A. Intriligator and C. Vafa, "A large N duality via a geometric transition," Nucl. Phys. B 603, 3 (2001) arXiv:hep-th/0103067.

[11] J.D. Edelstein, K. Oh and R. Tatar, "Orientifold, geometric transition and large $N$ duality for SO/Sp gauge theories," JHEP 0105, 009 (2001) arXiv:hep-th/0104037.

[12] K. Dasgupta, K. Oh and R. Tatar, "Geometric transition, large $N$ dualities and MQCD dynamics," Nucl. Phys. B 610, 331 (2001) arXiv:hep-th/0105066;

"Open/closed string dualities and Seiberg duality from geometric transitions in Mtheory," arXiv:hep-th/0106040;

"Geometric transition versus cascading solution," JHEP 0201, 031 (2002) arXiv:hepth/0110050].

[13] F. Cachazo, K. A. Intriligator and C. Vafa, "A large N duality via a geometric transition," Nucl. Phys. B 603, 3 (2001) arXiv:hep-th/0103067.

[14] F. Cachazo, B. Fiol, K. A. Intriligator, S. Katz and C. Vafa, "A geometric unification of dualities," Nucl. Phys. B 628, 3 (2002) arXiv:hep-th/0110028.

[15] F. Cachazo and C. Vafa, " $\mathrm{N}=1$ and $\mathrm{N}=2$ geometry from fluxes," arXiv:hepth/0206017.

[16] H. Ooguri and C. Vafa, "Worldsheet derivation of a large N duality," arXiv:hepth/0205297. 
[17] M. Aganagic and C. Vafa, " $G_{2}$ manifolds, mirror symmetry, and geometric engineering," arXiv:hep-th/0110171.

[18] D. E. Diaconescu, B. Florea and A. Grassi, "Geometric transitions and open string instantons," arXiv:hep-th/0205234.

[19] M. Aganagic, M. Marino and C. Vafa, "All loop topological string amplitudes from Chern-Simons theory," arXiv:hep-th/0206164.

[20] D. E. Diaconescu, B. Florea and A. Grassi, "Geometric transitions, del Pezzo surfaces and open string instantons," arXiv:hep-th/0206163.

[21] S. Kachru, S. Katz, A. E. Lawrence and J. McGreevy, "Open string instantons and superpotentials," Phys. Rev. D 62, 026001 (2000) arXiv:hep-th/9912151.

[22] M. Bershadsky, S. Cecotti, H. Ooguri and C. Vafa, "Kodaira-Spencer theory of gravity and exact results for quantum string amplitudes," Commun. Math. Phys. 165, 311 (1994) arXiv:hep-th/9309140.

[23] E. Witten, "Chern-Simons gauge theory as a string theory," arXiv:hep-th/9207094.

[24] M. Bertola, B. Eynard, J. Harnad, "Partition functions for Matrix Models and Isomonodromic Tau functions," arxiv:nlin.SI/0204054.

[25] G. Akemann, "Higher genus correlators for the Hermitian matrix model with multiple cuts," Nucl. Phys. B 482, 403 (1996) [arXiv:hep-th/9606004].

[26] S. R. Das and A. Jevicki, "String Field Theory And Physical Interpretation Of D = 1 Strings," Mod. Phys. Lett. A 5, 1639 (1990).

[27] A. Morozov, "Matrix Models as Integrable Systems," arxiv:hep-th/9502091.

[28] S. H. Shenker, "The Strength Of Nonperturbative Effects In String Theory," in Proceedings Cargese 1990, Random surfaces and quantum gravity, 191-200.

[29] M. Bershadsky, W. Lerche, D. Nemeschansky and N. P. Warner, "Extended N=2 superconformal structure of gravity and W gravity coupled to matter," Nucl. Phys. B 401, 304 (1993) arXiv:hep-th/9211040

[30] R. Dijkgraaf, "Intersection theory, integrable hierarchies and topological field theory," in Cargese Summer School on New Symmetry Principles in Quantum Field Theory 1991, arXiv:hep-th/9201003.

[31] A.B. Givental, "Gromov-Witten invariants and quantization of quadratic hamiltonians," arXiv:math.AG/0108100.

[32] A. Okounkov and R. Pandharipande, "Gromov-Witten theory, Hurwitz theory, and completed cycle," arXiv:math.AG/0204305.

[33] D. Ghoshal and C. Vafa, "c=1 string as the topological theory of the conifold," Nucl. Phys. B 453, 121 (1995) [arXiv:hep-th/9506122].

[34] R. Dijkgraaf and C. Vafa, to appear.

[35] P.B. Wiegmann and A. Zabrodin, "Conformal maps and integrable hierarchies," arXiv:hep-th/9909147.

[36] S. Y. Alexandrov, V. A. Kazakov and I. K. Kostov, "Time-dependent backgrounds of 2D string theory," arXiv:hep-th/0205079. 